\title{
CONSEQUENCES OF A SEXTUPLE-PRODUCT IDENTITY
}

\author{
JOHN A. EWELL \\ Department of Mathematical Sciences \\ Northern Illinois University \\ DeKalb, Illinois 60115
}

(Received March 28, 1986)

ABSTRACT. A sextuple-product identity, which essentially results from squaring the classical Gauss-Jacobi triple-product identity, is used to derive two trigonometrical identities. Several special cases of these identities are then presented and discussed.

KEY WORDS AND PHRASES. Sextuple-product identity, triple-product identity. 1980 AMS SUBJECT CLASSIFICATION CODE. O5A19.

1. INTRODUCTION.

The purpose of this paper is to prove the following theorem.

THEOREM 1. If $\theta \in R, \chi \in C$ and $|\chi|<1$, then

$$
\begin{aligned}
& 2 x \prod_{1}^{\infty}\left(1-x^{4 n}\right)^{2}\left(1-2 x^{4 n} \cos \theta+x^{u n}\right)^{2} \\
& \quad \times\left\{1-8 \sin ^{2}(\theta / 2) \sum_{1}^{\infty} \frac{k x^{4 k}}{1-x^{4 k}} \cos k \theta\right\} \\
&= \sum_{-\infty}^{\infty}(2 n+1)^{2} x^{(2 n+1)^{2}} \sum_{-\infty}^{\infty} x^{(2 n)^{2}} \cos 2 n \theta \\
&-\sum_{-\infty}^{\infty}(2 n)^{2} x^{(2 n)^{2}} \sum_{-\infty}^{\infty} x^{(2 n+1)^{2}} \cos (2 n+1) \theta
\end{aligned}
$$

and

$$
\begin{aligned}
& 4 \prod_{1}^{\infty}\left(1-x^{4 n}\right)^{2}\left(1-2 x^{4 n-2} \cos \theta+x^{8 n-4}\right)^{2} \cdot \sum_{1}^{\infty} \frac{k x^{2 k}}{1-x^{4 k}} \cos k \theta \\
&=\sum_{-\infty}^{\infty}(2 n+1)^{2} x^{(2 n+1)^{2}} \sum_{-\infty}^{\infty} x^{(2 n+1)^{2}} \cos (2 n+1) \theta \\
&-\sum_{-\infty}^{\infty}(2 n)^{2} x^{(2 n)^{2}} \sum_{-\infty}^{\infty} x^{(2 n)^{2}} \cos 2 n \theta
\end{aligned}
$$


The details of the proof are supplied in Section 2 where the major tool is the identity

$$
\begin{aligned}
& \prod_{1}^{\infty}\left(1-x^{2 n}\right)^{2}\left(1+a x^{2 n-1}\right)^{2}\left(1+a^{-1} x^{2 n-1}\right)^{2} \\
& \quad=\sum_{-\infty}^{\infty} x^{2 n^{2}} \sum_{-\infty}^{\infty} x^{2 n^{2}} a^{2 n}+x \sum_{-\infty}^{\infty} x^{2 n(n+1)} \sum_{-\infty}^{\infty} x^{2 n(n+1)} a^{2 n+1},
\end{aligned}
$$

valid for complex numbers $a, x$ such that $a \neq 0$ and $|\chi|<1$. In the papers [1] and [2] the author derives this identity in two different ways from the classical Gauss-Jacobi triple-product identity

$$
\prod_{1}^{\infty}\left(1-x^{2 n}\right)\left(1+a x^{2 n-1}\right)\left(1+a^{-1} x^{2 n-1}\right)=\sum_{-\infty}^{\infty} x^{n^{2}} a^{n}
$$

with the same restrictions on $a$ and $\chi$. For the first of these derivations a substantial assist by Gauss [3, pp. 78-79] is acknowledged. We shall also require the following identities

$$
\begin{aligned}
& \left\{(1 / 4) \cot (\theta / 2)+\sum_{1}^{\infty} \frac{x^{k} \sin k \theta}{1-x^{k}}\right\}^{3} \\
& =\{(1 / 4) \cot (\theta / 2)\}^{2}+\sum_{1}^{\infty} \frac{x^{k} \cos k \theta}{\left(1-x^{k}\right)^{2}}+\frac{1}{2} \sum_{1}^{\infty} \frac{k x^{k}}{1-x^{k}}(1-\cos k \theta), \\
& 2\left\{\sum_{1}^{\infty} \frac{x^{k}}{1-x^{2 k}} \sin k \theta\right\}^{2} \\
& =\sum_{1}^{\infty} \frac{k x^{2 k}}{1-x^{2 k}}+\sum_{1}^{\infty} \frac{x^{k}\left(1+x^{2 k}\right)}{\left(1-x^{2 k}\right)^{2}} \cos k \theta-\sum_{1}^{\infty} \frac{k x^{k}}{1-x^{2 k}} \cos k \theta .
\end{aligned}
$$

(In (1.5) $\theta$ is assumed to not be an even multiple of $\pi_{0}$ ) Identity (1.5) is due to Ramanujan [4,p. 139]. However, in [5] the author deduces both of these results from the triple-product identity (1.4). In Section 3 a few special cases of (1.1) and (1.2) are noted.

2. PROOF OF THEOREM 1 .

To prove (1.1) we first of all, let $x \rightarrow x^{2}$, $a \rightarrow-a x^{2}$ in (1.3), and multiply the resulting identity by ax to get

$$
\begin{aligned}
\left(a^{\frac{1}{2}}-a^{-\frac{1}{2}}\right)^{2} x \prod_{1}^{\infty}\left(1-x^{4 n}\right)^{2}\left(1-a x^{4 n}\right)^{2}\left(1-a^{-1} x^{4 n}\right)^{2} \\
=\sum_{-\infty}^{\infty} x^{(2 n)^{2}} \sum_{\infty}^{\infty} x^{(2 n+1)^{2}} a^{2 n+1}-\sum_{-\infty}^{\infty} x^{(2 n+1)^{2}} \sum_{-\infty}^{\infty} x^{(2 n)^{2} a^{2 n}} .
\end{aligned}
$$

Now, for an arbitrary complex variable $z$ regard $z D_{z}$ as an operator, $D_{z}$ denoting derivation with respect to $z$. Then, letting $F(a, x)$ denote the left side 
of (2.1), and putting $\alpha(x):=\sum x^{(2 n)^{2}}, \quad B(x):=\sum x^{(2 n+1)^{2}}$ (for both sums summation extending over $Z$ ), we have

$$
\left(a, D_{a}\right)^{2} F(a, x)=\alpha(x) \sum_{-\infty}^{\infty}(2 n+1)^{2} x^{(2 n+1)^{2}} a^{2 n+1}-\beta(x) \sum_{-\infty}^{\infty}(2 n)^{2} x^{(2 n)^{2}} a^{2 n},
$$

while

$$
\begin{aligned}
\left(x D_{x}\right) F(a, x)= & \alpha(x) \sum_{-\infty}^{\infty}(2 n+1)^{2} x^{(2 n+1)^{2}} a^{2 n+1}-\beta(x) \sum_{-\infty}^{\infty}(2 n)^{2} x^{(2 n)^{2}} a^{2 n} \\
& +x \alpha^{\prime}(x) \sum_{-\infty}^{\infty} x^{(2 n+1)^{2}} a^{2 n+1}-x \beta^{\prime}(x) \sum_{-\infty}^{\infty} x^{(2 n)^{2}} a^{2 n}
\end{aligned}
$$

Hence,

$$
\begin{array}{rl}
\left(a D_{a}\right)^{2} & F(a, x)-\left(x D_{x}\right) F(a, x) \\
& =-x \alpha^{\prime}(x) \sum_{-\infty}^{\infty} x^{(2 n+1)^{2}} a^{2 n+1}+x \beta^{\prime}(x) \sum_{-\infty}^{\infty} x^{(2 n)^{2}} a^{2 n} .
\end{array}
$$

For $k \in Z^{+}$and $t \in C,|t|<1$, put $u_{k}=u_{k}(t):=t^{k}\left(1-t^{k}\right)^{-1}$. Then by straightforward logarithmic differentiation, we get:

$$
\begin{gathered}
\left(a D_{a}\right)^{2} F(a, x)=\left\{\left(\frac{a^{\frac{1}{2}}+a^{-\frac{1}{2}}}{a^{\frac{1}{2}}-a^{-\frac{1}{2}}}-2 \sum_{1}^{\infty} u_{k}\left(x^{4}\right)\left(a^{k}-a^{-k}\right)\right)^{2}\right. \\
\left.-\frac{2}{\left(a^{\frac{1}{2}}-a^{\frac{-1}{2}}\right)^{2}}-2 \sum_{1}^{\infty} k u_{k}\left(x^{4}\right)\left(a^{k}+a^{-k}\right)\right\} F(a, x), \\
\left(x D_{x}\right) F(a, x)=\left\{1-8 \sum_{1}^{\infty} k u_{k}\left(x^{4}\right)-8 \sum_{1}^{\infty} u_{k}\left(x^{4}\right)\left[u_{k}\left(x^{4}\right)+1\right]\left(a^{k}+a^{-k}\right)\right\} F(a, x) .
\end{gathered}
$$

We now put $a:=e^{i \theta}$ and realize that $(2.2)$ becomes:

$$
\begin{aligned}
&-4 \sin ^{2}(\theta / 2) x \prod_{1}^{\infty}\left(a-x^{4 n}\right)^{2}\left(a-2 x^{4 n} \cos \theta+x^{8 n}\right)^{2} \\
&=\left\{-\sum_{-\infty}^{\infty}(2 n)^{2} x^{(2 n)^{2}} \sum_{-\infty}^{\infty} x^{(2 n+1)^{2}} \cos (2 n+1) \theta\right. \\
&\left.+\sum_{-\infty}^{\infty}(2 n+1)^{2} x^{(2 n+1)^{2}} \sum_{-\infty}^{\infty} x^{(2 n)^{2}} \cos (2 n) \theta\right\} \\
& \times\left\{-16\left((1 / 4) \cot (\theta / 2)+\sum_{1}^{\infty} u_{k}\left(x^{4}\right) \sin k \theta\right)^{2}-\frac{1}{2}+\frac{1}{2} \cot ^{2}(\theta / 2)\right. \\
&\left.-4 \sum_{1}^{\infty} k u_{k}\left(x^{4}\right) \cos k \theta+8 \sum_{1}^{\infty} k u_{k}\left(x^{4}\right)+16 \sum_{1}^{\infty} u_{k}\left(x^{4}\right)\left[u_{k}\left(x^{4}\right)+1\right] \cos k \theta\right\}^{-1} .
\end{aligned}
$$

By Ramanujan's identity (1.5) the foregoing identity then reduces to identity (1.1). To prove (1.2) we begin by transforming (1.3) under the substitutions $a \rightarrow-a$, 
$x \rightarrow x^{2}$. The remaining details are similar to the ones for the proof of (1.1), but in the final simplification identity (1.6) plays the same role as did identity (1.5) in the foregoing derivation.

3. SPECIAL CASES OF IDENTITIES $(1.1),(1.2)$.

COROLLARY 2. For each complex number $\chi$ such that $|x|<1$,

$$
\begin{aligned}
& \prod_{1}^{\infty}\left(1-x^{n}\right)^{6}=\sum_{0}^{\infty}(2 n+1)^{2} x^{n(n+1)} \sum_{-\infty}^{\infty} x^{n^{2}}-\sum_{1}^{\infty}(2 n)^{2} x^{n^{2}} \sum_{-\infty}^{\infty} x^{n(n+1)} \\
& 4 \prod_{1}^{\infty}\left(1-x^{4 n}\right)^{2}\left(1-x^{4 n-2}\right)^{4} \cdot \sum_{1}^{\infty} \frac{k x^{2 k}}{1-x^{4 k}} \\
& =\sum_{-\infty}^{\infty}(2 n+1)^{2} x^{(2 n+1)^{2}} \sum_{-\infty}^{\infty} x^{(2 n+1)^{2}}-\sum_{-\infty}^{\infty}(2 n)^{2} x^{(2 n)^{2}} \sum_{-\infty}^{\infty} x^{(2 n)^{2}}
\end{aligned}
$$

PROOF. To prove (3.1) we appeal to (1.1), putting $\theta=0$, dividing by $2 x$ and letting $x \rightarrow x^{1}$. To prove (3.2) put $\theta=0$ in (1.2).

COROLLARY 3. For each complex number $x$ such that $|x|<1$,

$$
\begin{gathered}
2 x \prod_{1}^{\infty}\left(1-x^{4 n}\right)^{2}\left(1+x^{8 n}\right)^{2}\left\{1-8 \sum_{1}^{\infty} \frac{(-1)^{j} j x^{8 j}}{1-x^{8 j}}\right\} \\
=\sum_{-\infty}^{\infty}(2 n+1)^{2} x^{(2 n+1)^{2}} \sum_{-\infty}^{\infty}(-1)^{n} x^{(2 n)^{2}} \\
4 \prod_{1}^{\infty}\left(1-x^{4 n}\right)^{2}\left(1+x^{8 n-4}\right)^{2} \sum_{1}^{\infty} \frac{(-1)^{j} 2 j x^{4 j}}{1-x^{8 j}} \\
=-\sum_{-\infty}^{\infty}(2 n)^{2} x^{(2 n)^{2}} \sum_{-\infty}^{\infty}(-1)^{n} x^{(2 n)^{2}} .
\end{gathered}
$$

PROOF. In (1.1) and (2,1) put $\theta=\pi / 2$ to respectively obtain (3.3) and (3.4). COROLLARY 4. For each complex number $\chi$ such that $|x|<1$,

$$
\begin{aligned}
& 2 x \prod_{1}^{\infty}\left(1-x^{4 n}\right)^{2}\left(1+x^{4 n}\right)^{4}\left\{1-8 \sum_{1}^{\infty} \frac{(-1)^{k} k x^{4 k}}{1-x^{4 k}}\right\} \\
& =\sum_{-\infty}^{\infty}(2 n+1)^{2} x^{(2 n+1)^{2}} \sum_{-\infty}^{\infty} x^{(2 n)^{2}}+\sum_{-\infty}^{\infty}(2 n)^{2} x^{(2 n)^{2}} \sum_{-\infty}^{\infty} x^{(2 n+1)^{2}} \\
& 4 \prod_{-\infty}^{\infty}\left(1-x^{4 n}\right)^{2}\left(1+x^{4 n-2}\right)^{4} \sum_{1}^{\infty} \frac{(-1)^{k} k x^{2 k}}{1-x^{4 k}} \\
& =-\sum_{-\infty}^{\infty}(2 n+1)^{2} x^{(2 n+1)^{2}} \sum_{-\infty}^{\infty} x^{(2 n+1)^{2}}-\sum_{-\infty}^{\infty}(2 n)^{2} x^{(2 n)^{2}} \sum_{-\infty}^{\infty} x^{(2 n)^{2}} .
\end{aligned}
$$

PROOF. Put $\theta=\pi$ in (1.1) and (1.2) to respectively obtain (3.5) and (3.6). 
REMARK. Of the six identities in the foregoing corollaries(3.1.)'nas been by far the most fruitful. For example in [1] the author has used the identity to prove Ramanujan's theorem on the divisibility of certain values of the partition function by the modulus 7. The identity was also used by the author in [6] to establish Jacobi's formula for the number of representations of a natural number by sums of four squares. However, the remaining identities certainly have some intrinsic interest, since each of them combines in a single expression infinite products, ordinary power series and "Lambert" series.

\section{REFERENCES}

1. EWELL, J.A. Completion of a Gaussian Derivation, Proc. Amer. Math. Soc. 84(1982), 311-314.

2. EWELL, J.A. Some Combinatorial Identities and Arithmetical Applications, Rocky Mountain J. of Math. 15 (2) (1985), 365-370.

3. MACMAHON, P.A. Combinatory Analysis, vo1. 2, Che1sea, New York, 1960.

4. RAMANUJAN, S. Collected Papers, Chelsea, New York, 1962.

5. EWELL, J.A. Consequences of the Triple- and Quintuple-product Identities, Houston J. of Math. (to appear)

6. EWELL, J.A. A Simple Derivation of Jacobi's Four-square Formula, Proc. Amer. Math. Soc. 85 (1982), 323-326. 


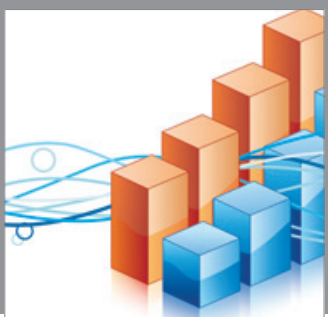

Advances in

Operations Research

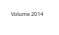

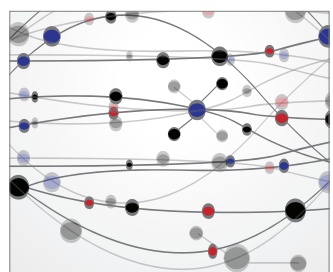

\section{The Scientific} World Journal
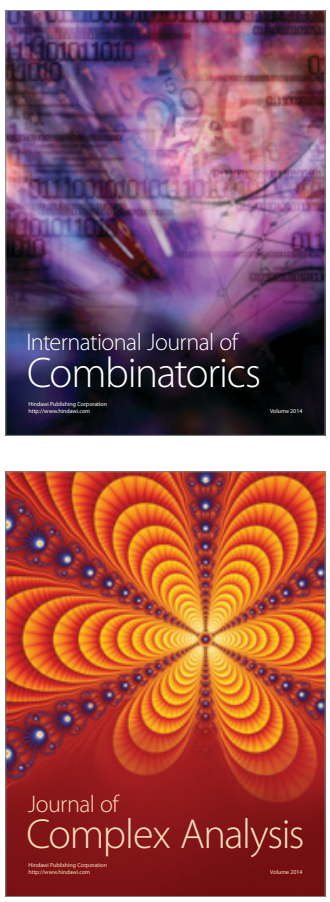

International Journal of

Mathematics and

Mathematical

Sciences
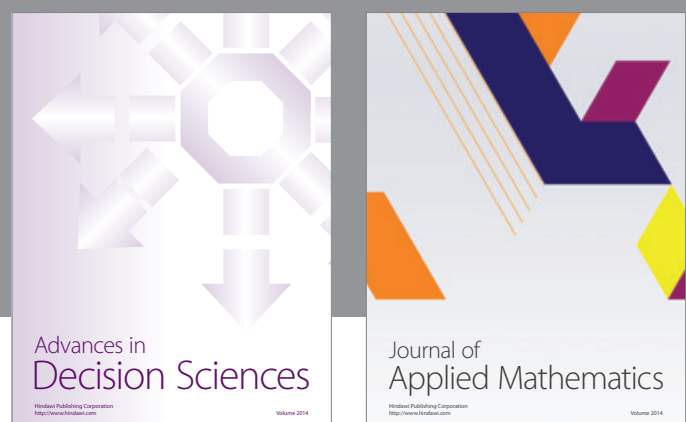

Journal of

Applied Mathematics
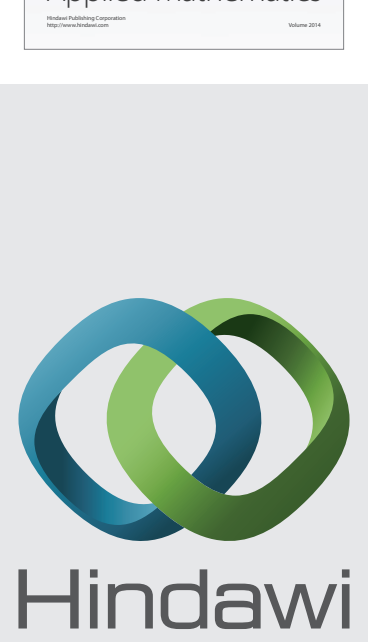

Submit your manuscripts at http://www.hindawi.com
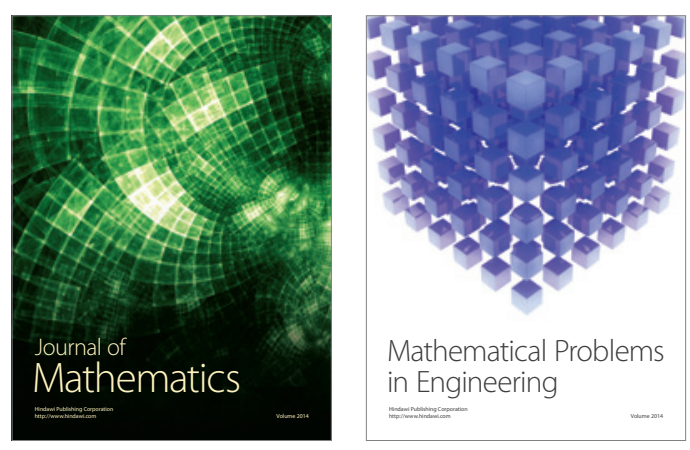

Mathematical Problems in Engineering
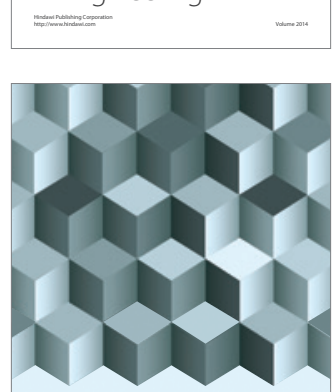

Journal of

Function Spaces
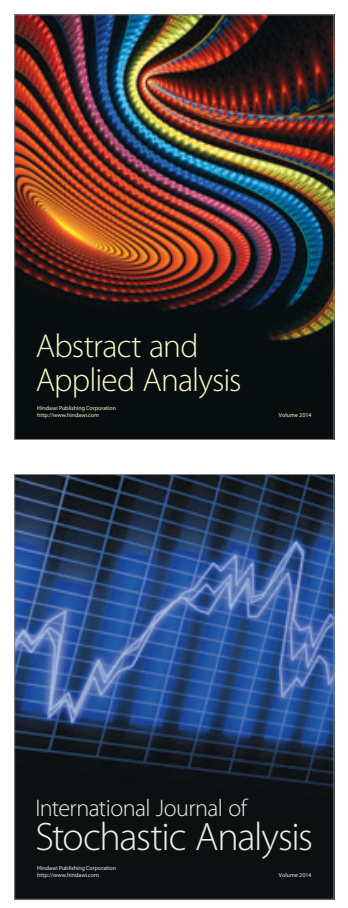

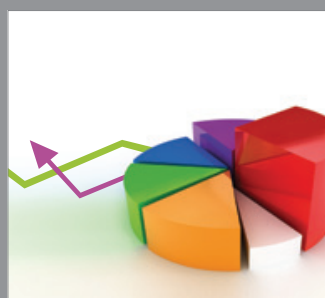

ournal of

Probability and Statistics

Promensencen
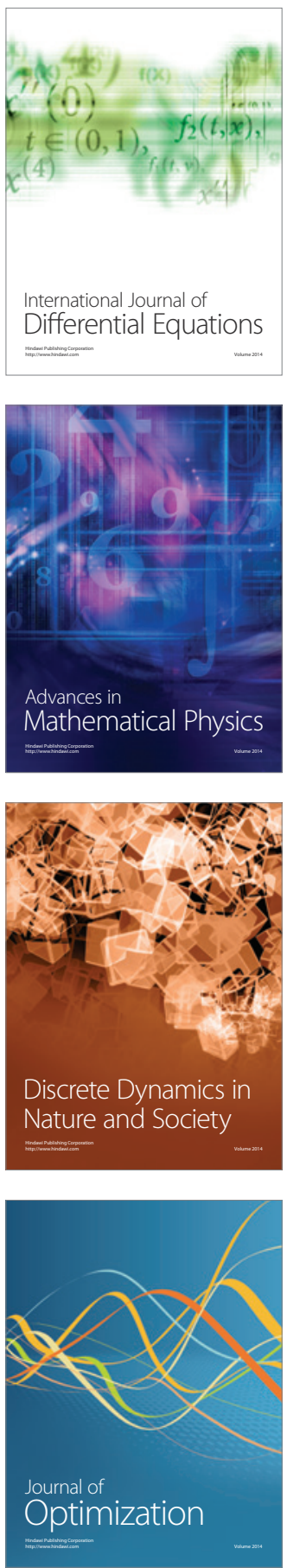